\title{
MEKK3-MEK5-ERK5 signaling promotes mitochondrial degradation
}

\author{
Jane E. Craig $\mathbb{0}^{1,2}$, Joseph N. Miller ${ }^{1,2}$, Raju R. Rayavarapu1, Zhenya Hong ${ }^{3,4}$, Gamze B. Bulut ${ }^{3}$, Wei Zhuang ${ }^{1}$, \\ Sadie Miki Sakurada ${ }^{1}$, Jamshid Temirov ${ }^{1}$, Jonathan A. Low ${ }^{5}$, Taosheng Chen $\mathbb{E}^{5}$, Shondra M. Pruett-Miller', \\ Lily Jun-shen Huang ${ }^{3}$ and Malia B. Potts (1) ${ }^{1}$
}

\begin{abstract}
Mitochondria are vital organelles that coordinate cellular energy homeostasis and have important roles in cell death. Therefore, the removal of damaged or excessive mitochondria is critical for maintaining proper cellular function. The PINK1-Parkin pathway removes acutely damaged mitochondria through a well-characterized mitophagy pathway, but basal mitochondrial turnover occurs via distinct and less well-understood mechanisms. Here we report that the MEKK3-MEK5-ERK5 kinase cascade is required for mitochondrial degradation in the absence of exogenous damage. We demonstrate that genetic or pharmacological inhibition of the MEKK3-MEK5-ERK5 pathway increases mitochondrial content by reducing lysosome-mediated degradation of mitochondria under basal conditions. We show that the MEKK3-MEK5-ERK5 pathway plays a selective role in basal mitochondrial degradation but is not required for nonselective bulk autophagy, damage-induced mitophagy, or restraint of mitochondrial biogenesis. This illuminates the MEKK3-MEK5-ERK5 pathway as a positive regulator of mitochondrial degradation that acts independently of exogenous mitochondrial stressors.
\end{abstract}

\section{Introduction}

Maintaining a population of healthy mitochondria is vital for proper development and is necessary to preserve tissue and organ function in response to stress conditions ${ }^{1}$. Mitochondria are well known for their importance in metabolism and energy production, but they are also a major source of reactive oxygen species (ROS), cytosolic DNA, and pro-apoptotic proteins ${ }^{2-5}$. Therefore, it is important to maintain sufficient quantities of healthy mitochondria to meet current metabolic demand, but dangerous to carry an excessive surplus of these organelles. Damaged or dysfunctional mitochondria tend to generate higher levels of ROS and cytosolic DNA compared to healthy mitochondria ${ }^{2,3,6}$. Exposure to high levels

\footnotetext{
Correspondence: Malia B. Potts (malia.potts@stjude.org)

${ }^{1}$ Department of Cell and Molecular Biology, St. Jude Children's Research Hospital, Memphis, Tennessee 38105, USA

${ }^{2}$ Integrated Biomedical Sciences Program, University of Tennessee Health

Science Center, Memphis, Tennessee 38163, USA

Full list of author information is available at the end of the article

Edited by Maria Victoria Niklison Chirou
}

of ROS can cause DNA mutations in both nuclear and mitochondrial DNA and can induce protein misfolding throughout the cell ${ }^{2,3}$. Mutations in mitochondrial DNA further decrease the health of the mitochondrial population and promote additional ROS production ${ }^{7-9}$. Thus, to maintain homeostasis, the cell has evolved complex systems for the quality control of mitochondria ${ }^{10}$. These systems are designed to balance the elimination of dysfunctional or superfluous mitochondria with mitochondrial biogenesis ${ }^{1}$. There are certain situations in which mitochondrial content changes even in the absence of damage, such as during differentiation of highly specialized cell types or induction of a metabolic shift towards a glycolytic phenotype or an oxidative phosphorylation phenotype $\mathrm{e}^{11-14}$.

A major mechanism of mitochondrial degradation is known as mitophagy, a form of selective autophagy ${ }^{10}$. Autophagy is an evolutionarily conserved process that uses double membrane vesicles known as autophagosomes to sequester cellular components such as damaged or excessive organelles and protein aggregates ${ }^{15}$. Autophagosomes then 
fuse with highly acidic lysosomes that contain hydrolases, which degrade the components inside ${ }^{15,16}$. Mitophagy follows the same steps as autophagy, but the autophagosome selectively forms around mitochondria that have been tagged for degradation by selective autophagy receptors such as NIX, OPTN, or $\mathrm{p} 62 / \mathrm{SQSTM} 1^{10}$. These proteins function by binding both the cargo and ATG8-family proteins (commonly referred to as "LC3" proteins) that decorate the forming autophagosome ${ }^{17}$.

Mitophagy plays important roles in development and in disease prevention. Insufficient mitochondrial clearance impairs erythroid maturation and can result in anemia ${ }^{18}$. Removal of paternal mitochondria after zygote formation increases fitness, as mitochondrial DNA is at increased risk of ROS damage during the process of spermatogenesis and fertilization ${ }^{19}$. Parkinson's disease, the second most common neurodegenerative disorder, is characterized by dysfunctional mitochondria and compromised mitophagy within the affected neurons ${ }^{20-22}$. In addition, dysfunctional mitochondria can contribute to the metabolic reprogramming of cancer cells ${ }^{23}$. Understanding how novel regulatory pathways contribute to the maintenance of mitochondrial homeostasis in different tissues could provide insights into the development of targeted treatments when these systems fail in disease.

Early insights into the mechanistic basis of mitochondrial degradation arose from studies in yeast, but how mitochondrial degradation is controlled in multicellular organisms such as humans is not yet fully understood ${ }^{24-26}$. Pink1-Parkin is the most well studied mitophagy regulatory pathway in animals. Although it has been very well characterized, most studies depend on ectopic Parkin overexpression and mitochondrial depolarization with carbonyl cyanide m-chlorophenyl hydrazine (CCCP) or other pharmacological agents ${ }^{20,27,28}$. Endogenous Parkin is poorly expressed in several mammalian tissues and cell lines, and it is clear that mitophagy can occur independently of Parkin and PINK $1^{29,30}$.

Here we report the identification of a previously undescribed role for the MEKK3-MEK5 signaling pathway as a regulator of basal mitophagy in cultured mammalian cells. MEKK3 and MEK5 are upstream activators of the extracellular signal-regulated kinase 5 (ERK5), which is the most recently discovered member of the mitogen-activated protein kinase (MAPK) family of proteins ${ }^{31}$. ERK5 is ubiquitously expressed in mammalian tissues and is activated by extracellular signals such as growth factors, several cellular stressors, and oxidative phosphorylation ${ }^{31-33}$. Mouse genetic studies have demonstrated that MEKK2/3-MEK5ERK5 signaling is required for early embryogenesis, development of the vasculature and muscles, endothelial cell function, and cardio protection ${ }^{31,32,34,35}$. Alterations in the MEKK2/3-MEK5-ERK5 pathway have been associated with several human diseases and disorders including cancer, childhood obesity, scoliosis, and cerebral cavernous malformation $^{36-39}$. Our studies indicate that MEKK3-MEK5ERK5 signaling is critical for basal mitochondrial degradation in mammalian cells and plays a relevant role in erythrocyte maturation.

\section{Materials and methods \\ Cell culture}

Transformed MEFs, U2OS cells stably expressing mitochondrial-targeted mCherry, U2OS cells stably expressing GFP-LC3B, and HeLa YFP-Parkin cells were received from Michael White's laboratory. The U2OS GFP-LC3B cells were originally a kind gift from Xiaodong Wang and the HeLa YFP-Parkin cells were originally a kind gift from Richard Youle. Mito-mKeima stably expressing U2OS cells were generated by transfection

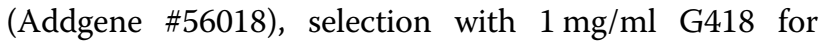
2 weeks, and flow sorted for high expressers. Cells were grown in Dulbecco's modified Eagle's medium (DMEM) supplemented with $10 \%$ fetal bovine serum (FBS), L-Glutamine, Penicillin and Streptomycin, and Sodium Pyruvate, and at $37{ }^{\circ} \mathrm{C}$ and $5 \% \mathrm{CO}_{2}$. Mito-mCherry, GFPLC3B, and mito-mKeima stable cells were maintained in $1 \mathrm{mg} / \mathrm{ml}$ G418 DMEM media.

\section{CRISPR/Cas9-knockout cell lines}

SQSTM1/p62- and MAPK7/ERK5-knockout U2OS cells were generated using CRISPR-Cas9 technology. Briefly, 400,000 U2OS cells were transiently transfected with precomplexed ribonuclear proteins consisting of $100 \mathrm{pmol}$ of chemically modified single-guide RNA (sgSQSTM1 5'-gagggaaagggcuugcaccg-3' or sgMAPK7 5'agacggcgaggacggcucug- $3^{\prime}$, Synthego), 35 pmol of Cas 9 protein (St. Jude Protein Production Core), and $200 \mathrm{ng}$ of pMaxGFP (Lonza) via nucleofection (Lonza, 4D-Nucleofector $^{\mathrm{TM}} \mathrm{X}$-unit) using solution P3 and program CM-104 in a small $(20 \mu \mathrm{l})$ cuvette according to the manufacturer's recommended protocol.

Five days post nucleofection, cells were single-cell sorted by fluorescence-activated cell sorting to enrich for GFP+ (transfected) cells, clonally selected, and verified for the desired targeted modification via targeted deep sequencing using gene-specific primers with partial Illumina adapter overhangs (hSQSTM1.F 5'-acagccccaca gtgacgacagaggg- $3^{\prime}$ and hSQSTM1.R $5^{\prime}$-tgggggaggaattagca gagcggca-3', or hMAPK7.F $5^{\prime}$-agctagtctgccacgaaccagccg c-3' and hMAPK7.R 5'-cgggcggaggacaccactccatagg- $3^{\prime}$, overhangs not shown). Next Generation Sequencing analysis of clones was performed using CRIS.py ${ }^{40}$. Genotypes of each clone are available upon request.

\section{Inhibitor treatments}

Unless otherwise stated, cells were treated with $10 \mu \mathrm{M}$ BIX02189 (Tocris 4842) and $10 \mu \mathrm{M} \mathrm{XMD8-92} \mathrm{(Tocris}$ 
4132) for $16 \mathrm{~h}$ and with $50 \mathrm{nM}$ Bafilomycin A1 (Sigma B1793) for $2 \mathrm{~h}$.

\section{Immunoblotting}

Western blottings were performed by lysing cells in $2 \times$ Laemmli loading buffer with $10 \mathrm{mM}$ dithiothreitol, separated on polyacrylamide gels, transferred to polyvinylidene difluoride membranes, and probed with antibodies against p62/SQSTM1 (CS 51145), XPB (SC-293), TOMM40 (MBL 3740), B-actin (Sigma A1978), VDAC (CS 4867S), S6K (CS 9202S), ERK5 (CS 3372S), mtCOX2 (Ab797393), MEKK3 (SC-28769), MEK5 (Invitrogen PA5-15083), PGC1 $\alpha$ (CS 2178S), and pERK5 (CS 3371S).

\section{Mitochondrial content}

Relative mitochondrial content was assessed either qualitatively by western blotting for endogenous mitochondrial proteins and non-mitochondrial loading controls (described above) or via quantitative analysis of fluorescently labeled mitochondria via flow cytometry. Mitochondria were fluorescently labeled either by stable expression of mitochondrial-targeted mCherry (mitomCherry) or staining with MitoTracker Green FM (Thermo Fisher, M7514). U2OS cells stably expressing mito-mCherry were plated in six-well plates for flow cytometry analysis or in Nunc Lab-Tek II chambered coverglass (Thermo, 155382) for microscopy imaging. Alternatively, cells were stained with $100 \mathrm{nM}$ MitoTracker Green FM for $30 \mathrm{~min}$ then washed with phosphatebuffered saline (PBS) three times. Cells were then trypsinized and assayed as described in the Flow Cytometry section below. Mitochondrial content per cell was measured from 10,000 cells per condition by quantifying the fluorescence of either mito-mCherry or MitoTracker Green FM, and the median value for each condition was normalized to the median value from the appropriate control condition.

\section{Mitochondrial uncoupling}

HeLa YFP-Parkin cells were treated with $10 \mathrm{nM} \mathrm{CCCP}$ overnight in addition to indicated treatments. Cells were then fixed and stained with 4',6-diamidino-2-phenylindole (DAPI) and TOMM20 antibody.

\section{Confocal imaging}

Regular confocal images were taken with Leica SP8 TCS equipped with White Light Laser, $405 \mathrm{~nm}$ diode laser using $\times 63 / 1.4 \mathrm{NA} / \mathrm{Oil} / \mathrm{HC}$ PL APO CS2/0.14 mm objective confocal microscope. Mito-mCherry images were created from Z-stacks taken at software calculated Nyquest pixel resolution. Higher resolution confocal images were taken using $\times 100 / 1.4 \mathrm{NA}$ objective with oversampled XY pixel size of $16 \mathrm{~nm}$ and Z-step size of $100 \mathrm{~nm}$ which were deconvolved using Huygens
Professional 16.10 (Scientific Volume Imaging, The Netherlands).

GFP-LC3B puncta and SQSTM1/p62 puncta were manually counted on a per cell basis

MAPK7/ERK5 wild-type and MAPK7/ERK5-knockout U2OS cells stably expressing mitochondrial-targeted mKeima were plated in Nunc Lab-Tek II chambered coverglass and imaged live on the Leica SP8 TCS confocal microscope. Data were analyzed by comparing 561 (acidic) signal to 458 (neutral) signal.

\section{Lysosomal acidification}

Lysosomal content was determined by staining with LysoTracker Red DND-99 (L7528) at $100 \mathrm{nM}$ for $30 \mathrm{~min}$. Lysosomal acidification was determined by staining with LysoSensor Green DND-189 (L7528) at $1 \mathrm{mM}$ for $10 \mathrm{~min}$. Cells were then imaged using a BioTek Cytation 5 Cell Imaging Multi-Mode Reader. Cells were imaged using $\times 20$ objective. Signal was analyzed via relative fluorescence using CellProfiler ${ }^{41}$.

\section{Flow cytometry}

Cells were treated as indicated, trypsinized, spun down at $400 \mathrm{rpm}$, and resuspended in buffer made from $1 \times$ PBS, $2 \%$ serum, $1 \mathrm{mM}$ EDTA, and $0.1 \%$ Sodium Azide. Cells were stained with DAPI. Fluorescence was then quantified on a BD Biosciences Fortessa by gating on single live cells and subsequently analyzed using FloJo v10.6.2.

\section{RNA sequencing}

RNA was extracted from cultured cells using RNeasy Kit (QIAGEN \#74104) following manufacturers' protocols. RNA concentration was measured using a NanoDrop (Thermo Fisher Scientific, Waltham, MA) and the quality of RNA was determined with a bioanalyzer (Agilent Technologies, Santa Clara, CA). Libraries were prepared using the TruSeq Stranded Total RNA Library Prep Kit (Illumina, San Diego, CA) and subjected to 100 cycle paired-end sequencing on the Illumina HiSeq platform.

\section{Promega autophagy assay}

HEK293 autophagy reporter cells (HiBiT-HaloTag-LC3, Promega \#GA1040) were grown in in DMEM (Thermo Fisher \#31053-028) supplemented with 10\% FBS (Hyclone \#SH30071), L-Glutamine (Thermo Fisher \#25030-081), Penicillin and Streptomycin (Thermo Fisher \#15140-122), Sodium Pyruvate (Thermo Fisher \#11360-070), and $500 \mu \mathrm{g} / \mathrm{mL}$ G418 (Thermo Fisher \#10131) at $37^{\circ} \mathrm{C}$ and $5 \%$ $\mathrm{CO}_{2}$. Cells (2500) in $25 \mu \mathrm{l}$ of G418-free media were plated into each well of Corning 8804BC 384-well plates and grown for $18 \mathrm{~h}$. Cells were then treated with ten-point curves with threefold dilutions and a top concentration of $60 \mu \mathrm{M}$ of Bafilomycin A1 (positive control for autophagy 
inhibition), Rapamycin (TOCRIS \#1292; positive control for autophagy activation), BIX02188, BIX02189, and XMD8-92, or dimethylsulfoxide (DMSO) using a V\&P Scientific S100 pin-tool before they were returned to $37^{\circ} \mathrm{C}$ with $5 \% \mathrm{CO}_{2}$ for $24 \mathrm{~h}$. The assay plates were then equilibrated to room temperature for $15 \mathrm{~min}$ before the addition of $25 \mu \mathrm{l}$ of Nano-Glo HiBiT Lytic Detection System (Promega \#N3030). The plates were then shaken at 300 r.p.m. for $2 \mathrm{~min}$ on an orbital shaker, incubated for $30 \mathrm{~min}$ at room temperature, and the luminescence of each well measured using a Perkin Elmer Envision plate reader.

\section{Erythroblast maturation}

Ter119-negative erythroid progenitors were isolated from embryonic day 13.5 or $14.5 \mathrm{Balb} / \mathrm{c}$ murine fetal livers as described ${ }^{42}$. The purity of the cells was confirmed by staining cells with PE-CD71 and allophycocyanin (APC)Ter119 antibodies (all from BD Biosciences) by flow cytometry. Approximately $10 \%$ of total fetal livers were purified as Ter119 negative. These progenitors were set up on retronectin coated plates for differentiation and were followed over three days for differentiation status and mitochondrial content. The data were acquired on a BD FACSCalibur and analyzed by FlowJo 8.8.4. To measure mitochondrial content, cells were stained with $25 \mathrm{nM}$ MitoTracker Deep Red FM (Molecular Probes, Invitrogen) for $30 \mathrm{~min}$ at $37^{\circ} \mathrm{C}$.

For inhibitor treatments starting on Day 0, Ter119negative progenitors were set up for differentiation directly after purification in erythropoietin (Epo) media in the presence of inhibitor on retronectin coated plates. For inhibitor treatments starting on Day 2 and Day 3 Ter119-negative progenitors, $20 \mathrm{~h}$ after culturing in Epo medium or 1 day after removal from EPO medium, were set up for differentiation separately, and inhibitors were added when indicated. Erythroid differentiation was monitored on days 1, 2, and 3 with PE-CD71 (BD Biosciences) and APC-Ter119 (eBioscience) by flow cytometry. 7-AAD (BD Biosciences) staining was used to exclude dead cells. Cells were treated with DMSO, $10 \mu \mathrm{M}$ BIX02188, or $10 \mu \mathrm{m}$ XMD8-92.

\section{Results}

SQSTM1/p62 constitutively delivers mitochondria to lysosomes for degradation in the absence of exogenous damage

The primary question we set out to address is what controls basal mitochondrial degradation in the absence of exogenous damage. This process is known to be mechanistically distinct from damage-induced mitophagy, which requires the kinase PINK1, the E3 ligase Parkin, and the selective autophagy receptors NDP52 and Optineurin $^{28,30}$. We first investigated whether an alternative selective autophagy receptor, SQSTM1/p62, supports mitochondrial degradation in the absence of exogenous damage. We selected p62 as an initial candidate because it cannot support damage-induced, Parkin-dependent mitophagy but has been reported to be important for mitochondrial degradation in other contexts ${ }^{17,28,43-45}$. We treated U2OS osteosarcoma cells with Bafilomycin A1 for $2 \mathrm{~h}$ to inhibit degradation of all cargo delivered to the lysosome and used immunofluorescent microscopy to determine whether such cargo included mitochondria and p62. Our experiments revealed that a minority of the mitochondrial population (marked by TOMM20) were delivered to LAMP1 (lysosomal associated membrane protein 1)-positive lysosomes in the absence of any mitophagy-inducing treatment, and all LAMP1-encapsulated mitochondria were co-labeled with p62 (Fig. 1a, b). In contrast, the vast majority of LAMP1-negative mitochondria were negative for p62 (Fig. 1a, b). In the absence of Bafilomycin treatment we observed rare instances of p62-positive mitochondria co-localized with LAMP1 (Fig. 1a).

To determine whether lysosomal activity detectably limited mitochondrial accumulation under basal conditions, we stably expressed a construct encoding the CoxVIII leader sequence fused to mCherry in U2OS cells (U2OS mito-mCherry cells) and measured mCherry fluorescent intensity per cell by flow cytometry as a marker of mitochondrial abundance. Short durations of lysosomal inhibition $(<5 \mathrm{~h})$ did not detectably increase bulk mitochondrial content per cell, whereas longer durations of lysosomal inhibition caused a steady increase of mitochondrial content over time (Fig. 1c). Together these results indicate that steady-state mitochondrial abundance is limited by ongoing delivery of a small subset of p62-labeled mitochondria to lysosomes for degradation. Treatment with Bafilomycin A1 (BafA1) increased total protein levels of p62 in a dose-dependent manner, confirming that p62 is continually degraded by the lysosome under basal conditions (Fig. 1d). Together, these results suggest that p62 labels mitochondria destined for delivery to lysosomes in the absence of exogenous damage or ectopic Parkin overexpression.

To test whether p62 is required for basal mitochondrial degradation, we depleted p62 by RNA interference (RNAi) or CRISPR/Cas9-mediated gene editing and asked whether total mitochondrial content per cell increased as a result. Multiple independent siRNA oligos targeting p62 caused an increase in mitochondrial accumulation in U2OS mito-mCherry cells (Fig. 1e and Supplementary Fig. 1A). Similarly, p62-knockout U2OS cells exhibited increased mitochondrial accumulation relative to parental U2OS cells as measured by MitoTracker Green FM staining (Fig. If and Supplementary Fig. 1B). Taken together, our data indicate p62 is important for basal delivery of mitochondria to lysosomes for degradation in the absence of exogenous damage. 
A
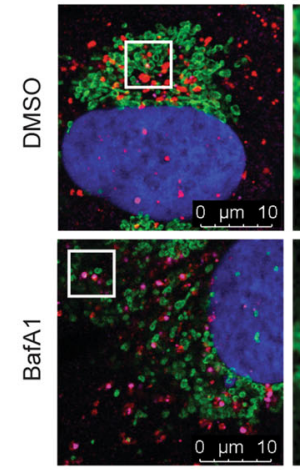

B

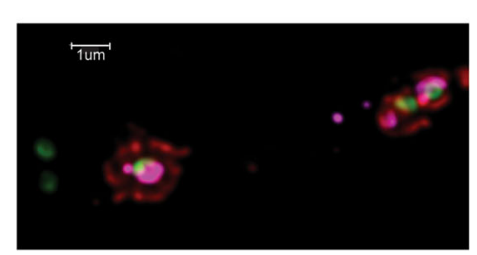

E

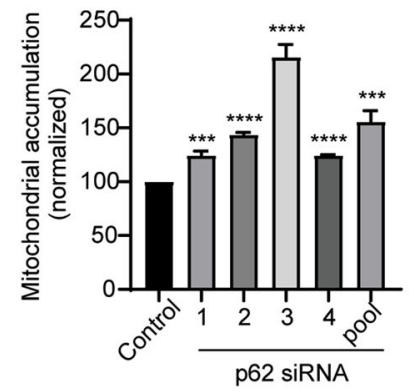

TOMM20

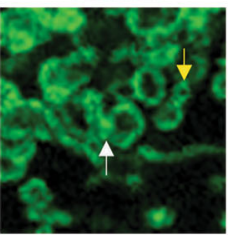

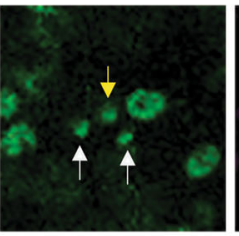

\section{C}

$\mathbf{F}$

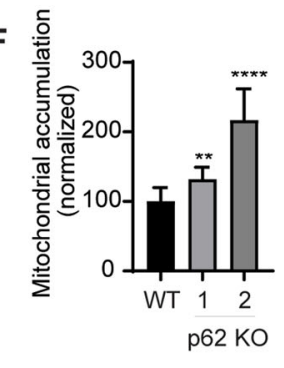

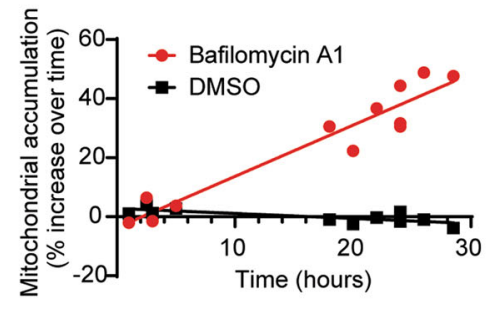

Merge
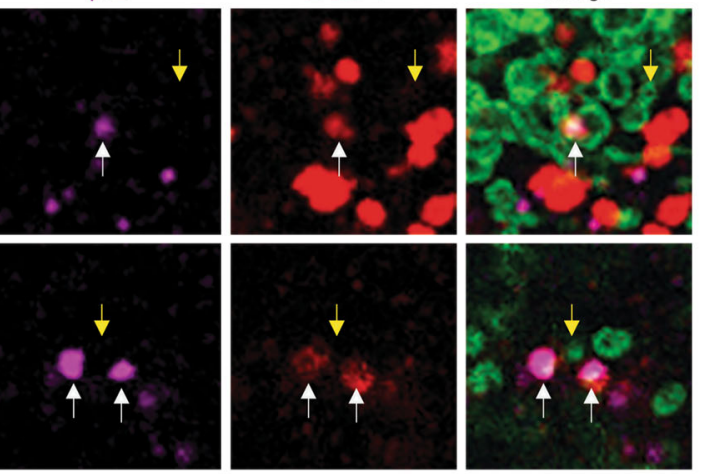

D

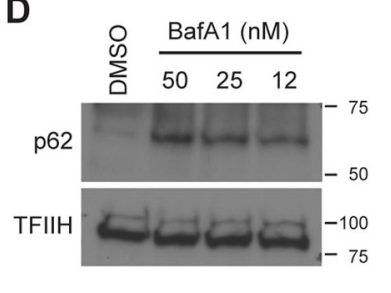

Fig. 1 SQSTM1/p62 constitutively delivers mitochondria to lysosomes for degradation in the absence of exogenous damage. a U2OS cells were treated with vehicle (DMSO) or with $50 \mathrm{nM}$ Bafilomycin $\mathrm{A} 1$ for $2 \mathrm{~h}$ to block degradation of lysosomal contents and analyzed by fluorescence confocal microscopy. Representative images are shown. TOMM20 (Green), p62 (purple), LAMP1 (red), Dapi (blue). White arrows show instances of mitochondria tagged by p62 and surrounded by lysosomes. Yellow arrows designate mitochondria that are not being degraded. b Representative high-resolution confocal image of cells treated with bafilomycin A1 as in a. Tomm20 (green), p62 (purple), LAMP1 (red). c U2OS mito-mCherry cells were treated with $50 \mathrm{nM}$ Bafilomycin A1 or vehicle (DMSO) for the indicated time. Mitochondrial accumulation was detected by flow cytometry and displayed as percent increase over time. $\mathbf{d}$ Protein levels of p62 from U2OS cells that were treated with the indicated concentrations of Bafilomycin A1 overnight were detected by western blotting analysis. TFIIH was used as a loading control. e U2OS mito-mCherry cells were transfected with the indicated siRNA oligos or siControl (the p62 Si pool contains oligos 1-4). Seventy-two hours later, mitochondrial accumulation was measured by flow cytometry. Mean \pm SE of $n=3$ independent experiments is shown; ${ }^{* * *} p<0.001 ;{ }^{* * * *} p<0.0001$. f p62-knockout U2OS cells were stained with 100 nM MitoTracker Green FM for 30 min, and mitochondrial accumulation was measured by flow cytometry. Mean \pm SE of $n=7$ independent experiments is shown; ${ }^{* *} p<0.01 ; * * * 0<0.0001$.

Nomination of putative mitophagy regulatory pathways

To identify novel mitophagy regulatory pathways, we utilized Functional Signature Ontology (FuSiOn), a method for unbiased identification of functionally related proteins, to nominate protein kinases from the human genome that function similarly to p62 under basal conditions ${ }^{46}$. FuSiOn identified ten kinases (BMP2K, DCLK3, LIMK2, MAP2K5/MEK5, MAP3K3/MEKK3, ROS1, SIK2, TAOK2, ULK1, and ULK2) whose depletion mimicked the phenotypic effect of p62 depletion in HCT116 colon cancer cells ${ }^{46}$. To determine whether any of these ten candidates promotes basal mitophagy, we depleted each kinase individually in U2OS mito-mCherry cells and measured the resulting change in average mitochondrial content per cell. Depletion of seven of the ten kinases caused an increase in mitochondrial content similar to that caused by depletion of p62 (Fig. 2a). Next, we depleted each kinase in U2OS GFP-LC3B cells and measured accumulation of GFP-LC3B as a marker of nonselective autophagy inhibition. Two candidates (MAP3K3 

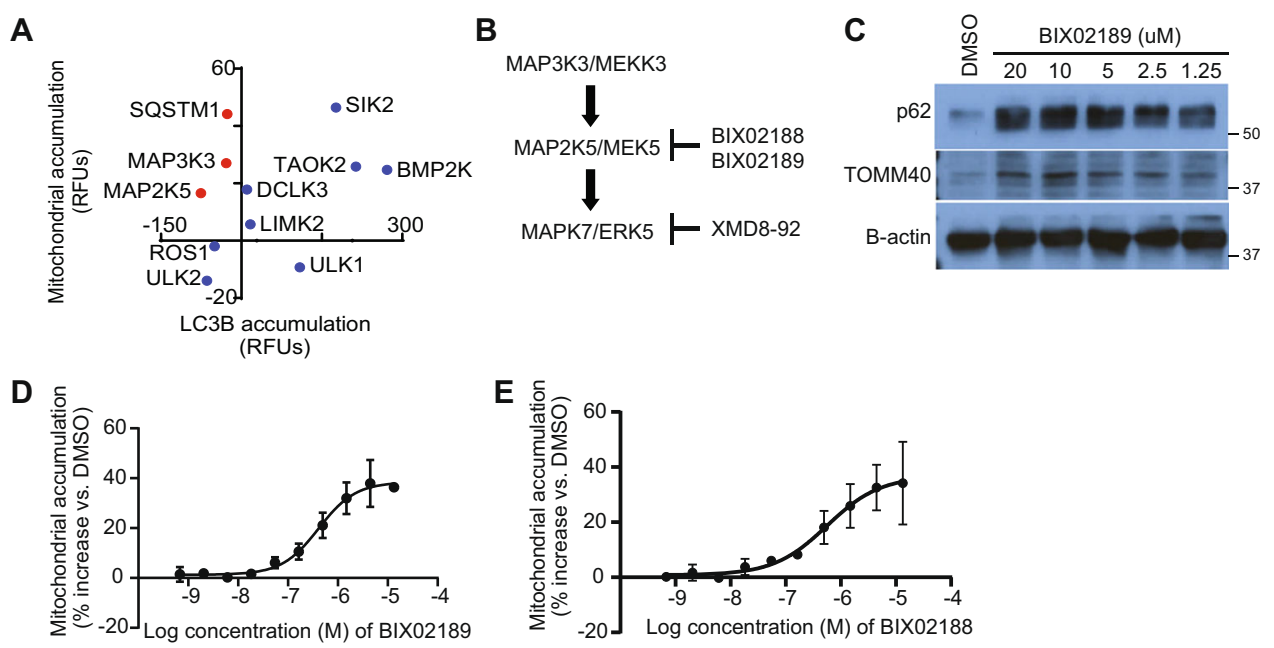

Fig. 2 Nomination of putative mitophagy regulatory pathways. a Candidate mitophagy-regulating genes identified by FuSiOn were screened by siRNA-mediated depletion in U2OS mito-mCherry cells and U2OS GFP-LC3B cells. Red dots denote selectivity of candidate gene for mitochondrial degradation. b Model of the MAP3K3 kinase cascade including inhibitors. c Protein levels of p62 and mitochondrial marker TOMM40 from mouse embryonic fibroblasts treated with vehicle (DMSO) or the indicated concentrations of the BIX02189 overnight. $\mathbf{d}$ Mitochondrial accumulation in U2OS mito-mCherry cells treated overnight with vehicle (DMSO) or BIX02189 was measured by flow cytometry. Mean \pm SD of $n=2$ independent experiments is shown. e Mitochondrial accumulation in U2OS mito-mCherry cells treated overnight with vehicle (DMSO) or BIX02188 was measured by flow cytometry. Mean \pm SD of $n=3$ independent experiments is shown.

and MAP2K5) phenocopied p62's selectivity for mitochondria, demonstrated by the relatively consistent LC3B levels in addition to the increase in mitochondrial content (Fig. 2a). MAP3K3 encodes MEKK3, which activates the MEK5-ERK5 kinase cascade. MAP2K5 encodes the MEKK3 substrate MEK5 (Fig. 2b). We confirmed ontarget efficacy of the siRNA pools directed against MAP3K3/MEKK3 and MAP2K5/MEK5 (Supplementary Fig. 1C). Maximum intensity projection images of U2OS mito-mCherry cells confirmed that the mito-mCherry signal remained localized to mitochondrial network after depletion of MEKK3 or MEK5 and demonstrated the accumulation of mitochondrial network in individual cells, supporting the flow cytometric data (Supplementary Fig. 1D). Next, we tested whether pharmacological inhibition of MEK5 kinase activity could alter mitochondrial abundance using two small molecule inhibitors of MEK5 (BIX02188 and BIX02189) ${ }^{47}$. Both BIX02188 and BIX02189 inhibited MEK5 activity and increased mitochondrial content in a dose-dependent manner in varied mammalian cells (Fig. 2c-e and Supplementary Fig. 1E). Given the structural similarity between the two inhibitors, we measured the off-target activities of BIX02188 and BIX02189 by Kinome Profiling and determined that the off-target activities do not overlap (Supplementary Table 1). This increases the likelihood that the observed increase in mitochondrial content is due to inhibition of the intended target, MEK5. Together, these results indicate that MEKK3-MEK5 signaling restrains mitochondrial accumulation.

\section{The MEKK3-MEK5-ERK5 kinase cascade prevents} accumulation of excess mitochondria

We proceeded to assay whether the canonical downstream component of this kinase cascade, ERK5 (encoded by the MAPK7 gene), also plays a role in restraining mitochondrial accumulation. RNAi-mediated depletion of ERK5 increased the average mitochondrial content per cell, indicating that ERK5 (like p62, MEKK3, and MEK5) prevents excess accumulation of mitochondria under basal conditions (Fig. 3a and Supplementary Fig. 1D). A small molecule inhibitor of ERK5, XMD8 $-92^{48}$, also increased mitochondrial content in a dose-dependent manner (Fig. 3b, c). Thus, we generated MAPK7/ERK5knockout U2OS cells using CRISPR/Cas9 technology and analyzed mitochondrial content via western blotting and MitoTracker Green FM staining. ERK5-knockout cells exhibited increased mitochondrial accumulation relative to the parental controls (Fig. 3d, e). These data indicate that the canonical MEKK3-MEK5-ERK5 kinase cascade restrains mitochondrial accumulation under basal conditions.

We asked whether the MEKK3-MEK5-ERK5 kinase cascade promotes mitochondrial degradation through regulation of p62 protein levels. MEKK3, MEK5, and p62 all contain PB1 domains, which mediate protein-protein dimerization $^{49}$. We hypothesized that MEKK3-MEK5ERK5 pathway inhibition might decrease p62 protein stability, or alternatively, might reduce p62 expression given that ERK5 is known to translocate to the nucleus and regulate gene transcription when activated ${ }^{31,50}$. 

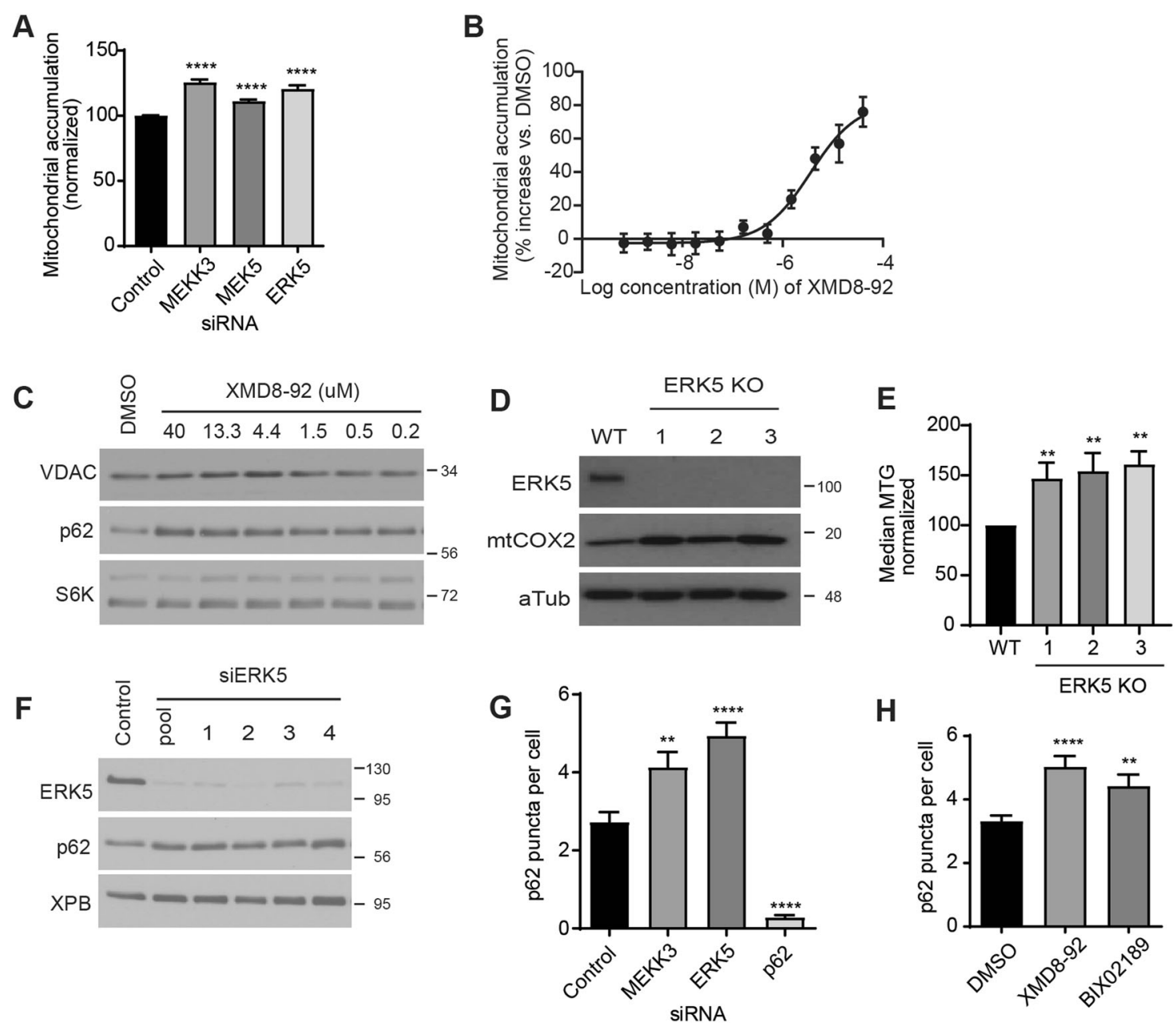

Fig. 3 The MEKK3-MEK5-ERK5 kinase cascade prevents accumulation of excess mitochondria. a U2OS mito-mCherry cells were transfected with the indicated siRNA oligos. Seventy-two hours later, mitochondrial accumulation was analyzed by flow cytometry. Mean \pm SD of $n=3$ independent experiments is shown. b, c Mitochondrial accumulation in U2OS mito-mCherry cells treated overnight with vehicle (DMSO) or XMD8-92 was measured by flow cytometry or western blot, respectively. Mean \pm SD of $n=2$ independent experiments is shown $\mathbf{b}$. $\mathbf{d}$, e Mitochondrial levels of three independent MAPK7/ERK5-knockout U2OS clones were assessed by western blotting analysis of mitochondrial marker mtCOX2 (d) or by MitoTracker Green FM staining and flow cytometry (e). f Protein levels of p62 from U2OS cells that were treated with ERK5 siRNA for $72 \mathrm{~h}$ were detected by western blotting analysis. XPB was used as a loading control. $\mathbf{g}, \mathbf{h}$ U2OS cells were either transfected with the indicated siRNAs for $72 \mathrm{~h}$ (g) or treated with the indicated drug at $10 \mu \mathrm{M}$ overnight (h). Cells were fixed and stained with p62 antibody and then imaged. See Supplementary Fig. 1f, g for representative images.

In either of these cases, a decrease in p62 levels upon MEKK3-MEK5-ERK5 pathway inhibition could explain why mitochondria accumulate under those conditions.

However, genetic or pharmacological inactivation of the MEKK3-MEK5-ERK5 kinase cascade induced p62 protein accumulation rather than decrease in abundance, indicating that MEKK3-MEK5-ERK5 signaling is not required for p62 expression or stability (Figs. 2c and 3c, f). Using immunofluorescent microscopy, we found that genetic or pharmacological inactivation of the MEKK3-MEK5-ERK5 kinase cascade caused an increase in the average number of cytoplasmic p62 puncta per cell (Fig. 3g, h and Supplementary Fig. 1F, G). The punctate accumulation of the selective autophagy adaptor protein p62 upon inhibition of the MEKK3-MEK5-ERK5 pathway is consistent with the interpretation that this pathway promotes one or more forms of selective autophagy under basal conditions.

\section{The MEKK3-MEK5-ERK5 pathway is required for lysosomal degradation of mitochondria}

To determine the underlying cause of increased mitochondrial content observed upon inhibition of MEKK3MEK5-ERK5 signaling, we considered and tested three distinct possibilities: (1) induction of mitochondrial biogenesis; (2) nonselective inhibition of the autophagylysosome pathway; and (3) selective inhibition of 
mitochondrial degradation. PGC1 $\alpha$ is a critical regulator of mitochondrial biogenesis ${ }^{51}$, and increased protein levels and activation of PGC1 $\alpha$ leads to an increase in transcription factors TFAM, TFB1M, and TFB2M ${ }^{52}$. Therefore, if inhibition of MEKK3-MEK5-ERK5 signaling increases mitochondrial abundance by inducing mitochondrial biogenesis, we should detect increased expression of PGC1 $\alpha$, TFAM, TFB1M, and TFB2M upon inhibition of the pathway. RNA sequencing confirmed that there was no increase in the mRNA levels of these major mitochondrial biogenesis-related transcription factors upon knockout of ERK5 (Fig. 4a and Supplementary Table 2). We also verified that the protein levels of the master mitochondrial biogenesis factor PGC1 $\alpha$ did not increase upon inhibition of the MEKK3-MEK5-ERK5 pathway. In fact, PGC1 $\alpha$ levels decreased upon pharmacological inhibition of MEK5 or ERK5 (Supplementary Fig. 2A), consistent with existing literature reporting a positive role for ERK5 signaling in promoting mitochondrial biogenesis ${ }^{35}$. Therefore, MEKK3-MEK5-ERK5 signaling does not restrain mitochondrial accumulation by limiting mitochondrial biogenesis.

In addition, MEK5/ERK5 signaling is not required for nonselective autophagy or for general lysosomal function. We performed a Promega Autophagy Assay, in which we treated HEK293 cells expressing the HiBiTLC3 autophagy reporter with a range of concentrations of DMSO, Bafilomycin A1, Rapamycin, BIX02188, BIX02189, and XMD8-92 for 24 h. As expected, Bafilomycin A1 effectively inhibited nonselective autophagy and Rapamycin effectively induced autophagy across wide dose ranges, as evidenced by increased (Bafilomycin) or decreased (Rapamycin) accumulation of the HiBiT-LC3 reporter (Fig. 4b). In contrast, BIX02188, BIX02189, and XMD8-92 did not inhibit nonselective autophagy even at concentrations as high as $60 \mu \mathrm{M}$ (Fig. 4b). Furthermore, we determined that inhibition of the MEK5-ERK5 pathway does not cause lysosomal deacidification or alter lysosomal content (Supplementary Fig. 2B, C). We also monitored autophagosome formation and autophagic flux using U2OS GFP-LC3B cells and observed no disruptions in either process upon treatment with XMD8-92 (Supplementary Fig. 2D, E).

The combined findings indicate the MEKK3-MEK5ERK5 kinase cascade restrains mitochondrial accumulation independent of mitochondrial biogenesis, driving bulk autophagy, or supporting general lysosome function. Next, we questioned whether the MEKK3-MEK5-ERK5 kinase cascade is required for selective degradation of mitochondria. Therefore, we developed WT and ERK5 KO U2OS cell lines expressing mitochondrial-targeted monomeric Keima, a pH-dependent fluorescent protein $^{53,54}$ to measure the delivery of mitochondria to lysosomes. When targeted to the mitochondria, the $\mathrm{pH}$-dependent fluorescence allows us to distinguish between mitochondria in the cytoplasm $(458 \mathrm{~nm})$ and mitochondria that are being degraded in the highly acidic lysosome $(561 \mathrm{~nm})^{55}$. We found that all three independent ERK5-knockout clones had significantly less mitochondria in the lysosomes compared to parental cells (Fig. 4c, d). Furthermore, in parental U2OS mito-mKeima cells, pharmacological inhibition of ERK5 using XMD8-92 decreased the amount of basal mitophagy compared to DMSO control (Fig. 4e). However, inhibition of the MEKK3-MEK5-ERK5 pathway by genetic or pharmacological tools did not mitigate CCCP-induced mitophagy either in the absence or presence of ectopic Parkin overexpression (Fig. 4f, g and Supplementary Fig. 2F-I). The combined data indicate the MEKK3-MEK5-ERK5 pathway specifically promotes lysosome-mediated mitochondrial degradation under basal conditions and is not required for nonspecific bulk autophagy, general lysosome function, or damage-induced mitophagy nor for restraint of mitochondrial biogenesis.

\section{MEKK3-MEK5-ERK5 pathway is required for differentiation and mitophagy in erythroid progenitors}

Previous studies suggest that ERK5-deficient cells develop altered nucleotide metabolism, impairing erythropoiesis in mice ${ }^{56}$. Erythropoiesis involves an ordered acquisition and loss of key differentiation markers ${ }^{57}$. As erythroid progenitors must eliminate their mitochondria to fully mature, we evaluated whether ERK5-regulated mitochondrial degradation participates in erythrocyte maturation ${ }^{58}$. To investigate the possible role of the MEKK3-MEK5-ERK5 pathway in erythrocyte maturation, Ter119-negative erythroid progenitors were isolated from murine fetal livers and induced to differentiate by addition of Epo ex vivo. We followed the expression of two specific differentiation markers: CD71, the transferrin receptor, whose expression decreases as erythroblasts mature, and Ter119, an antigen expressed on cell surfaces of more mature erythroblasts ${ }^{57}$. BIX02188 or vehicle control (DMSO) were present throughout the ex vivo differentiation process. Pharmacological inhibition of MEK5 impaired erythroid differentiation as evidenced by reduced percentages of cells reaching the most advanced Ter $119^{+} \mathrm{CD} 71^{\text {low }}$ stage of differentiation (Fig. 5a). Notably, MEK5 inhibition significantly increased basal mitochondrial content at all stages of differentiation (Fig. 5b). As differentiation progressed, both BIX02188-treated and DMSO-treated cells lost mitochondrial content over time; however, mitochondrial content was always significantly higher in BIX02188-treated cells relative to DMSOtreated cells at each stage (Fig. 5b). Similar results were observed when ERK5 was inhibited using XMD8-92 (Fig. 6a, b). Erythroid differentiation was similarly inhibited by genetic depletion of MEK5 using short hairpin RNA (Supplementary Fig. 3). Together, these data 


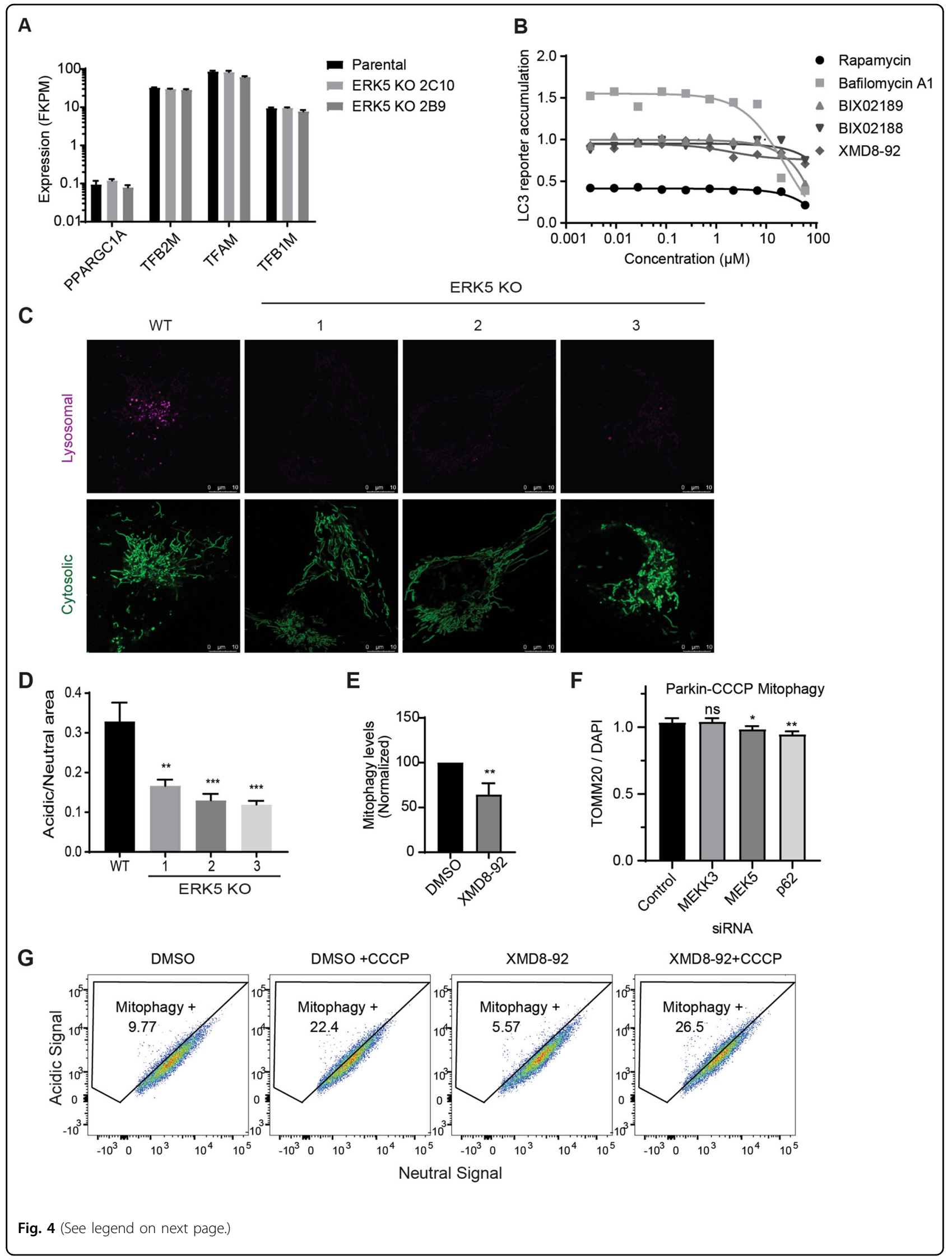


(see figure on previous page)

Fig. 4 The MEKK3-MEK5-ERK5 pathway is required for lysosomal degradation of mitochondria. a RNA-seq was performed on parental U2OS cells and two MAPK7/ERK5-knockout U2OS clones. Expression of several mRNAs encoding mitochondrial biogenesis genes is shown. $\mathbf{b}$ HEK293 cells stably expressing the HiBiT-LC3 bulk-autophagy reporter were treated with vehicle (DMSO) or the indicated compounds for $24 \mathrm{~h}$ in duplicate. Accumulation of the reporter was measured using the Promega Autophagy Assay system and normalized to vehicle-treated cells. $\mathbf{c}$, $\mathbf{d}$ Representative images (c) and quantification (d) of active mitophagy events in parental (WT) and ERK5-knockout U2OS cells expressing mitochondrial-targeted mKeima. e Mitophagy levels in parental U2OS cells expressing mitochondrial-targeted mKeima were assessed by flow cytometry and quantified. Cells were treated overnight with either DMSO or $10 \mu \mathrm{M}$ XMD8-92. Mean \pm SD of $n=3$ independent experiments is shown. $\mathbf{f}$ HeLa YFP-Parkin stable cells were transfected with the indicated siRNA for $72 \mathrm{~h}$ and treated with $10 \mu \mathrm{M} \mathrm{CCCP}$ overnight. Cells were fixed, stained with anti-Tom20 and DAPI, and imaged. Mitochondrial content was quantified and normalized to DAPI. g WT U2OS cells expressing mitochondrial-targeted mKeima were treated overnight with vehicle control, $10 \mu \mathrm{M}$ CCCP, $10 \mu \mathrm{M}$ XMD8-92, or both. Mitophagy levels were analyzed via flow cytometry.

A DMSO
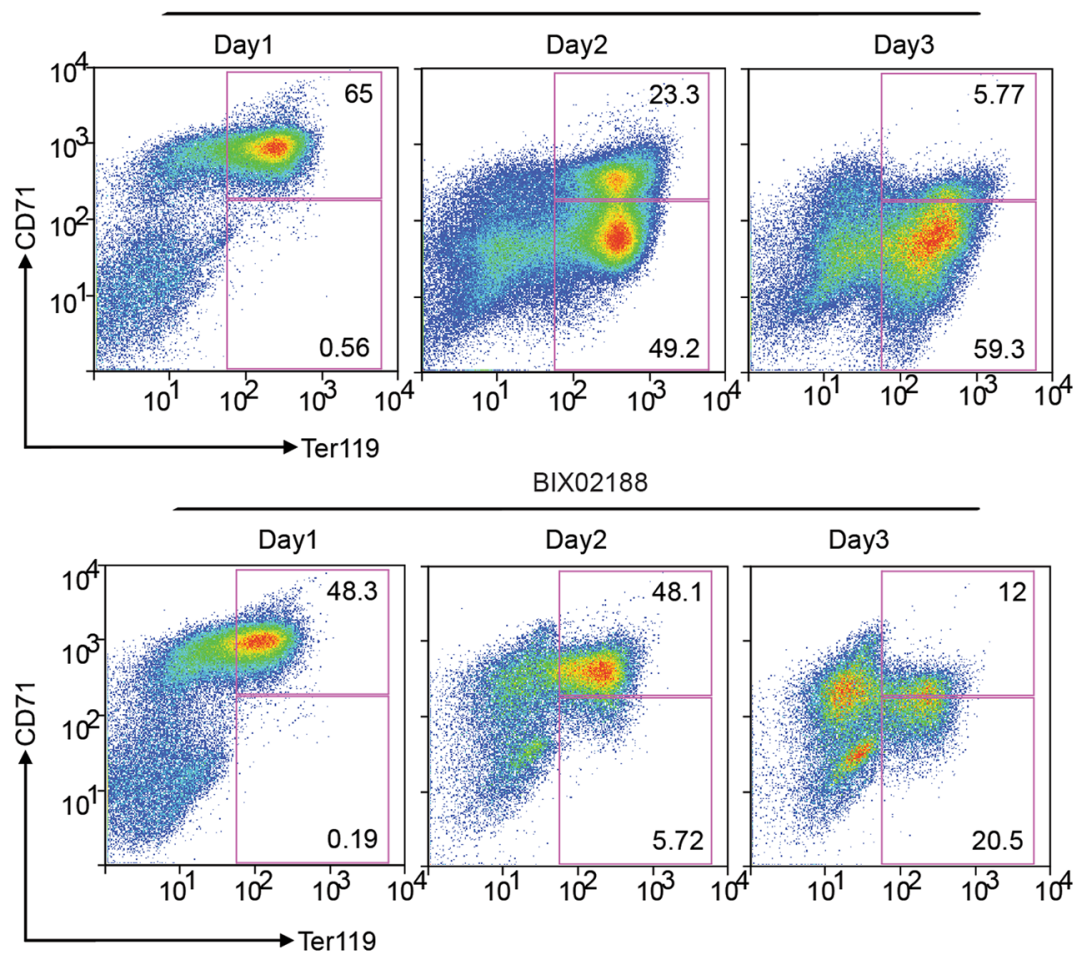

$\mathrm{B}$
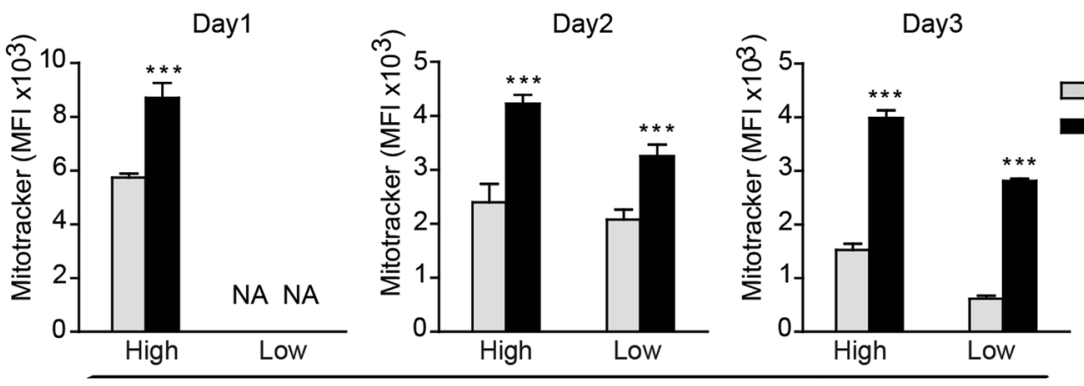

CD71 levels

Fig. 5 MEK5 is required for differentiation and mitophagy in erythroid progenitors. a Murine erythroid progenitors were treated with the BIX02188 (30 $\mu \mathrm{M})$ or vehicle control (DMSO) prior to initiation of differentiation. Levels of CD71 and Ter119 were measured by flow cytometry. Representative profiles are shown. b Mitochondrial mass was quantified in Ter $119^{+} \mathrm{CD} 71^{\text {high }}$ and Ter $119^{+} \mathrm{CD} 71^{\text {low }}$ erythroblasts using median fluorescence intensity (MFI) of MitoTracker Deep Red FM. The data represent three replicates. ${ }^{* * *} P<0.001$. 

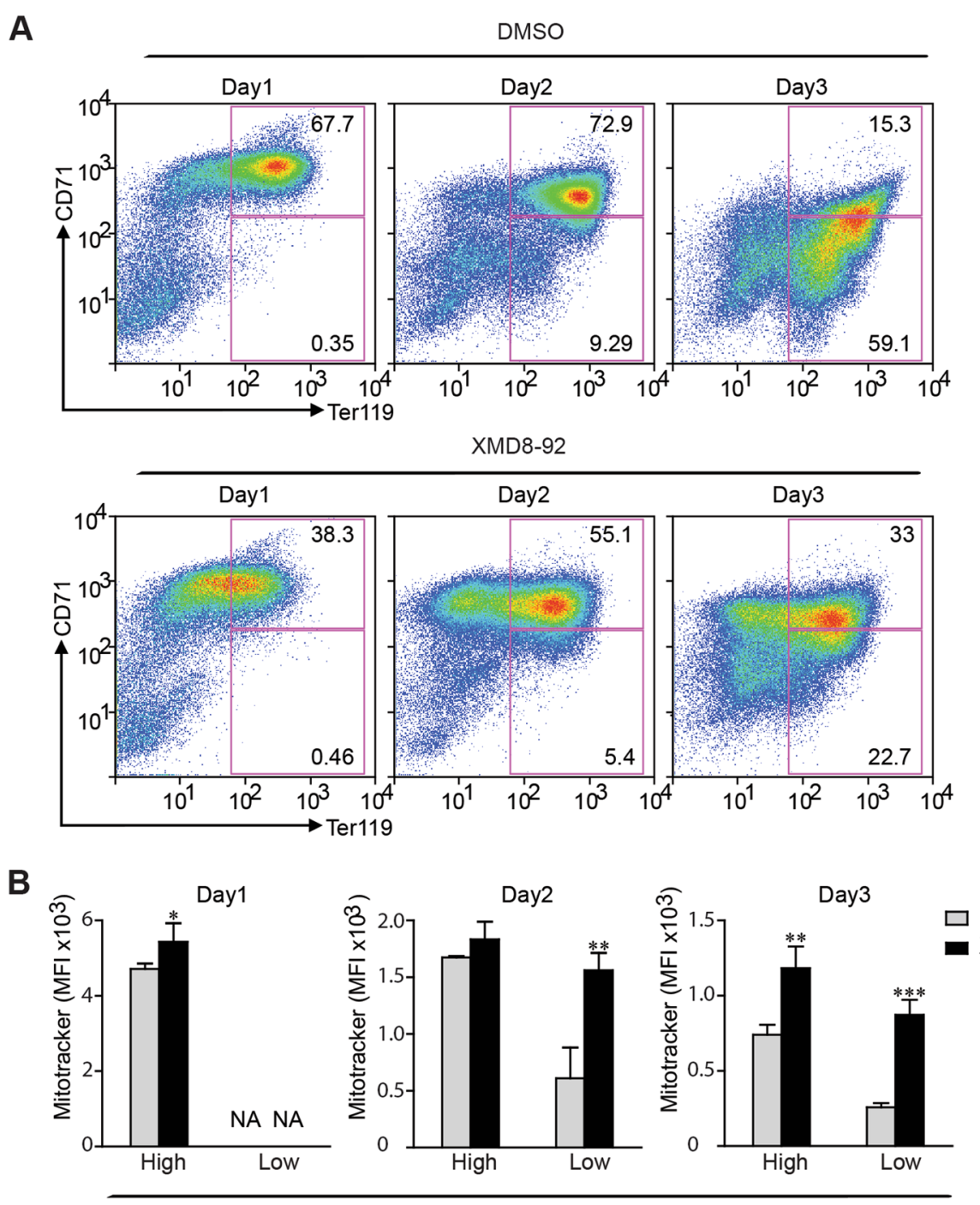

CD71 levels

Fig. 6 ERK5 is required for differentiation and mitophagy in erythroid progenitors. a Murine erythroid progenitors were treated with XMD8-92 $(5 \mu \mathrm{M})$ or vehicle control (DMSO) prior to initiation of differentiation. Levels of CD71 and Ter119 were measured by flow cytometry. Representative profiles are shown. b Mitochondrial mass was quantified in Ter $119^{+} \mathrm{CD} 71^{\text {high }}$ and Ter $119^{+} \mathrm{CD} 71^{\text {low }}$ erythroblasts using median fluorescence intensity (MFI) of MitoTracker Deep Red FM. The data represent three replicates. ${ }^{*} P<0.05$; ${ }^{*} P<0.01$; ${ }^{* *} P<0.001$.

indicate that MEK5-ERK5 signaling is required for proper erythroid differentiation and promotes clearance of mitochondria.

\section{Discussion}

Currently, there is substantial interest in the role autophagy plays in maintaining normal physiology and how dysfunctional mitophagy can lead to or enhance diseases. Our discovery of the MEKK3-MEK5-ERK5 pathway as a novel mitophagy regulator could facilitate a deeper understanding of how different mechanisms act to maintain homeostasis and prevent disease in different tissues. Using genetic and pharmacological inhibition of the MEKK3-
MEK5-ERK5 pathway, we observed mitochondrial content increase in human cancer cells, mouse embryonic fibroblasts, and primary erythroid progenitor cells isolated from the murine fetal liver. This suggests that MEKK3-MEK5ERK5 restrains mitochondrial accumulation in diverse cells and tissues. We also observed an increase in the levels of mitophagy receptor protein p62 after pharmacological or genetic inhibition of MEKK3-MEK5-ERK5 signaling. We confirmed that inhibition of pathway activity results in decreased lysosome-dependent degradation of mitochondria using mitochondrial-targeted mKeima, without affecting lysosome acidification or nonselective bulkautophagy levels in the cell. Taken together, we found 
that the MEKK3-MEK5-ERK5 pathway promotes mitophagy without the addition of exogenous mitochondrial damage, which could have great implication on studying mitophagy in the context of development and disease.

In addition, our findings provide evidence for the importance of p62 in mitophagy under basal conditions. We have found that SQSTM1/p62 routinely delivers mitochondria to lysosomes without the addition of exogenous damage. We have found that inhibition of autophagy non-specifically with Bafilomycin A1 increases total mitochondrial load in cells, indicating that mitophagy is an ongoing maintenance process. Whether MEKK3-MEK5-ERK5 signaling controls mitochondrial degradation by directly or indirectly regulating p62 remains to be determined, although we have ruled out the possibility that MEKK3-MEK5-ERK5 signaling is required for p62 expression or stability. Direct interactions between the PB1 domain of p62 and the PB1 domains of MEKK3 and MEK5 have been reported, providing a potential means for direct regulation of p62 by the MEKK3-MEK5-ERK5 kinase cascade ${ }^{49,59}$.

We found that basal mitochondrial degradation, which requires p62 and MEKK3-MEK5-ERK5 signaling, is quite distinct from damage-induced mitophagy, which does not require p62 or MEKK3-MEK5-ERK5. We sought to investigate whether developmentally programmed mitochondrial degradation in the erythrocyte lineage required MEK5ERK5 signaling as a well-established model of mitochondrial clearance in the absence of exogenous damage. Although impaired maturation of erythrocytes upon MEK5 or ERK5 inhibition precluded full assessment of the impact on mitochondrial turnover, the mitochondrial content was increased at each stage of erythroid maturation in MEK5ERK5 inhibited cells. Taken together with the lack of a requirement for MEKK3-MEK5-ERK5 signaling in CCCPinduced mitophagy, it appears that the MEKK3-MEK5ERK5 pathway may be solely designated for basal mitochondrial clearance. It is also possible that MEKK3-MEK5ERK5 signaling may be required for induction of mitochondrial degradation by other stimuli, such as statin treatment, hypoxia, or forced metabolic switch from glycolytic metabolism to oxidative phosphorylation ${ }^{60-63}$. These questions remain to be addressed in future work.

Although MEKK3-MEK5-ERK5 signaling has been implicated in many aspects of mammalian development and disease, it is also the most recently discovered MAP kinase pathway, and much work needs to be done to fully understand how the MEKK3-MEK5-ERK5 kinase cascade is acting. It will be interesting to determine whether any of the previously identified roles for the ERK5 pathway in development, physiology, or disease represent an underlying role for mitochondrial degradation. For example, ERK5 signaling is well established to play an anti-apoptotic role, and mitochondria harbor several pro-apoptotic molecules including cytochrome $c$, Smac/DIABLO, and $\mathrm{AIF}^{31,64,65}$. It is possible that active ERK5 signaling antagonizes autophagy in part through elimination of pro-apoptotic molecules via delivery of mitochondria to lysosomes for degradation. These and other interesting hypotheses arising from our current work remain to be tested in future studies.

\begin{abstract}
Acknowledgements
We thank M.A. White, F. Rivas, and M. Kundu for valuable discussions. We are grateful to H. Gan and M. Wang for their assistance. We wish to acknowledge support from the Center for Advanced Genome Engineering, Flow Cytometry and Cell Sorting Facility, Cell and Tissue Imaging Center, and Hartwell Center for Biotechnology. This study was supported in part by the National Institute of General Medical Sciences of the National Institutes of Health under Award Number R01 GM132231 (M.B.P.), National Natural Science Foundation of China 81873430 (Z.H.), the National Cancer Institute of the National Institutes of Health under Award Number P30 CA021765 (Charles Roberts), the American Lebanese Syrian Associated Charities (ALSAC), and St. Jude Children's Research Hospital.
\end{abstract}

\section{Author details}

'Department of Cell and Molecular Biology, St. Jude Children's Research Hospital, Memphis, Tennessee 38105, USA. IIntegrated Biomedical Sciences Program, University of Tennessee Health Science Center, Memphis, Tennessee 38163, USA. ${ }^{3}$ Department of Cell Biology, The University of Texas Southwestern Medical Center at Dallas, Dallas, Texas 75390, USA. ${ }^{4}$ Department of

Hematology, Tongji Hospital, Tongji Medical College, Huazhong University of Science and Technology, Wuhan, China. ${ }^{5}$ Department of Chemical Biology and Therapeutics, St. Jude Children's Research Hospital, Memphis, Tennessee 38105, USA

\section{Conflict of interest}

The authors declare that they have no conflict of interest.

\section{Publisher's note}

Springer Nature remains neutral with regard to jurisdictional claims in published maps and institutional affiliations.

The online version of this article (https://doi.org/10.1038/s41420-020-00342-7) contains supplementary material, which is available to authorized users.

Received: 21 July 2020 Revised: 9 September 2020 Accepted: 28 September 2020

Published online: 20 October 2020

\section{References}

1. Pickles, S., Vigie, P. \& Youle, R. J. Mitophagy and quality control mechanisms in mitochondrial maintenance. Curr. Biol. 28, R170-R185 (2018).

2. Murphy, M. P. How mitochondria produce reactive oxygen species. Biochem. J. 417, 1-13 (2009).

3. Kausar, S., Wang, F. \& Cui, H. The role of mitochondria in reactive oxygen species generation and its implications for neurodegenerative diseases. Cells $\mathbf{7}$, https:/doi.org/10.3390/cells7120274 (2018).

4. McArthur, K. et al. BAK/BAX macropores facilitate mitochondrial herniation and mtDNA efflux during apoptosis. Science 359, https://doi.org/10.1126/science. aao6047 (2018).

5. West, A. P. et al. Mitochondrial DNA stress primes the antiviral innate immune response. Nature 520, 553-557 (2015).

6. Sliter, D. A. et al. Parkin and PINK1 mitigate STING-induced inflammation. Nature 561, 258-262 (2018).

7. Hahn, A. \& Zuryn, S. Mitochondrial genome (mtDNA) mutations that generate reactive oxygen species. Antioxidants $\mathbf{8}$, https://doi.org/10.3390/antiox8090392 (2019).

8. Mattiazzi, M. et al. The mtDNA T8993G (NARP) mutation results in an impairment of oxidative phosphorylation that can be improved by antioxidants. Hum. Mol. Genet. 13, 869-879 (2004). 
9. Nissanka, N. \& Moraes, C. T. Mitochondrial DNA damage and reactive oxygen species in neurodegenerative disease. FEBS Lett. 592, 728-742 (2018).

10. Ding, W. X. \& Yin, X. M. Mitophagy: mechanisms, pathophysiological roles, and analysis. Biol. Chem. 393, 547-564 (2012).

11. Esteban-Martinez, L. et al. Programmed mitophagy is essential for the glycolytic switch during cell differentiation. EMBO J. 36, 1688-1706 (2017).

12. Esteban-Martinez, L. \& Boya, P. BNIP3L/NIX-dependent mitophagy regulates cell differentiation via metabolic reprogramming. Autophagy 14, 915-917 (2018).

13. Palikaras, K., Lionaki, E. \& Tavernarakis, N. Mechanisms of mitophagy in cellular homeostasis, physiology and pathology. Nat. Cell Biol. 20, 1013-1022 (2018).

14. $\mathrm{Hu}, \mathrm{C}$. et al. Energy metabolism plays a critical role in stem cell maintenance and differentiation. Int. J. Mol. Sci. 17, 253 (2016).

15. Mizushima, N. Autophagy: process and function. Genes Dev. 21, 2861-2873 (2007).

16. Dikic, I. Proteasomal and autophagic degradation systems. Annu. Rev. Biochem. 86, 193-224 (2017).

17. Yamada, T., Dawson, T. M., Yanagawa, T., lijima, M. \& Sesaki, H. SQSTM1/p62 promotes mitochondrial ubiquitination independently of PINK1 and PRKN/parkin in mitophagy. Autophagy, 1-7, https://doi. org/10.1080/15548627.2019.1643185 (2019).

18. Sandoval, H. et al. Essential role for Nix in autophagic maturation of erythroid cells. Nature 454, 232-235 (2008).

19. Sutovsky, P. et al. Ubiquitinated sperm mitochondria, selective proteolysis, and the regulation of mitochondrial inheritance in mammalian embryos. Biol. Reprod. 63, 582-590 (2000).

20. Narendra, D., Tanaka, A., Suen, D. F. \& Youle, R. J. Parkin is recruited selectively to impaired mitochondria and promotes their autophagy. J. Cell Biol. 183, 795-803 (2008).

21. Gao, F. et al. Mitophagy in Parkinson's disease: pathogenic and therapeutic implications. Front. Neurol. 8, 527 (2017).

22. Kalia, L. V. \& Lang, A. E. Parkinson's disease. Lancet 386, 896-912 (2015).

23. Gaude, E. \& Frezza, C. Defects in mitochondrial metabolism and cancer. Cancer Metab. 2, 10 (2014).

24. Chen, Y. \& Klionsky, D. J. The regulation of autophagy - unanswered questions. J. Cell Sci. 124, 161-170 (2011).

25. Nakatogawa, H., Suzuki, K., Kamada, Y. \& Ohsumi, Y. Dynamics and diversity in autophagy mechanisms: lessons from yeast. Nat. Rev. Mol. Cell Biol. 10, 458-467 (2009).

26. Glick, D., Barth, S. \& Macleod, K. F. Autophagy: cellular and molecular mechanisms. J. Pathol. 221, 3-12 (2010).

27. Drake, L. E., Springer, M. Z., Poole, L. P., Kim, C. J. \& Macleod, K. F. Expanding perspectives on the significance of mitophagy in cancer. Semin. Cancer Biol. 47, 110-124 (2017).

28. Lazarou, M. et al. The ubiquitin kinase PINK1 recruits autophagy receptors to induce mitophagy. Nature 524, 309-314 (2015).

29. Huynh, D. P., Dy, M., Nguyen, D., Kiehl, T. R. \& Pulst, S. M. Differential expression and tissue distribution of parkin isoforms during mouse development. Brain Res. Dev. Brain Res. 130, 173-181 (2001).

30. McWilliams, T. G. et al. Basal mitophagy occurs independently of PINK1 in mouse tissues of high metabolic demand. Cell Metab. 27, $439-449$ (2018). e435.

31. Nithianandarajah-Jones, G. N., Wilm, B., Goldring, C. E., Muller, J. \& Cross, M. J. ERK5: structure, regulation and function. Cell Signal 24, 2187-2196 (2012).

32. Nishimoto, S. \& Nishida, E. MAPK signalling: ERK5 versus ERK1/2. EMBO Rep. 7, 782-786 (2006).

33. Khan, A. U. H. et al. Mitochondrial Complex I activity signals antioxidant response through ERK5. Sci. Rep. 8, 7420 (2018).

34. Hayashi, M. et al. Targeted deletion of BMK1/ERK5 in adult mice perturbs vascular integrity and leads to endothelial failure. J. Clin. Invest. 113, 1138-1148 (2004).

35. Liu, W. et al. Metabolic stress-induced cardiomyopathy is caused by mitochondrial dysfunction due to attenuated Erk5 signaling. Nat. Commun. 8, 494 (2017).

36. Simoes, A. E., Rodrigues, C. M. \& Borralho, P. M. The MEK5/ERK5 signalling pathway in cancer: a promising novel therapeutic target. Drug Discov. Today 21, 1654-1663 (2016)

37. Zhu, $\mathrm{H}$. et al. Role of extracellular signal-regulated kinase 5 in adipocyte signaling. J. Biol. Chem. 289, 6311-6322 (2014).

38. Zhou, T. et al. Mutant MAPK7-induced idiopathic scoliosis is linked to impaired osteogenesis. Cell Physiol. Biochem. 48, 880-890 (2018).
39. Cullere, X., Plovie, E., Bennett, P. M., MacRae, C. A. \& Mayadas, T. N. The cerebral cavernous malformation proteins CCM2L and CCM2 prevent the activation of the MAP kinase MEKK3. Proc. Natl Acad. Sci. USA 112, 14284-14289 (2015).

40. Connelly, J. P. \& Pruett-Miller, S. M. CRIS.py: a versatile and high-throughput analysis program for CRISPR-based genome editing. Sci. Rep. 9, 4194 (2019).

41. McQuin, C. et al. CellProfiler 3.0: next-generation image processing for biology. PLoS Biol. 16, e2005970 (2018).

42. Sulahian, R., Cleaver, O. \& Huang, L. J. Ligand-induced EpoR internalization is mediated by JAK2 and p85 and is impaired by mutations responsible for primary familial and congenital polycythemia. Blood 113, 5287-5297 (2009).

43. Matsumoto, G., Shimogori, T., Hattori, N. \& Nukina, N. TBK1 controls autophagosomal engulfment of polyubiquitinated mitochondria through p62/ SQSTM1 phosphorylation. Hum. Mol. Genet. https://doi.org/10.1093/hmg/ ddv179 (2015).

44. Yamada, T. et al. Mitochondrial stasis reveals p62-mediated ubiquitination in Parkin-independent mitophagy and mitigates nonalcoholic fatty liver disease. Cell Metab. https://doi.org/10.1016/j.cmet.2018.06.014 (2018).

45. Nguyen, T. D. et al. Loss of the selective autophagy receptor p62 impairs murine myeloid leukemia progression and mitophagy. Blood 133, 168-179 (2019).

46. Potts, M. B. et al. Using functional signature ontology (FUSION) to identify mechanisms of action for natural products. Sci. Signal 6, ra90 (2013).

47. Tatake, R. J. et al. Identification of pharmacological inhibitors of the MEK5/ERK5 pathway. Biochem. Biophys. Res. Commun. 377, 120-125 (2008).

48. Yang, Q. et al. Pharmacological inhibition of BMK1 suppresses tumor growth through promyelocytic leukemia protein. Cancer Cell 18, 258-267 (2010).

49. Nakamura, K., Kimple, A. J., Siderovski, D. P. \& Johnson, G. L. PB1 domain interaction of p62/sequestosome 1 and MEKK3 regulates NF-kappaB activation. J. Biol. Chem. 285, 2077-2089 (2010).

50. Drew, B. A., Burow, M. E. \& Beckman, B. S. MEK5/ERK5 pathway: the first fifteen years. Biochim. Biophys. Acta 1825, 37-48 (2012).

51. Jornayvaz, F. R. \& Shulman, G. I. Regulation of mitochondrial biogenesis. Essays Biochem. 47, 69-84 (2010).

52. Villarroya, J., Giralt, M. \& Villarroya, F. Mitochondrial DNA: an up-and-coming actor in white adipose tissue pathophysiology. Obesity 17, 1814-1820 (2009).

53. Kogure, T., Kawano, H., Abe, Y. \& Miyawaki, A. Fluorescence imaging using a fluorescent protein with a large Stokes shift. Methods 45, 223-226 (2008).

54. Tantama, M., Hung, Y. P. \& Yellen, G. Imaging intracellular pH in live cells with a genetically encoded red fluorescent protein sensor. J. Am. Chem. Soc. 133, 10034-10037 (2011).

55. Biel, T. G. \& Rao, V. A. Mitochondrial dysfunction activates lysosomaldependent mitophagy selectively in cancer cells. Oncotarget 9, 995-1011 (2018).

56. Angulo-lbanez, M. et al. Erk5 contributes to maintaining the balance of cellular nucleotide levels and erythropoiesis. Cell Cycle 14, 3864-3876 (2015).

57. Koulnis, M. et al. Identification and analysis of mouse erythroid progenitors using the CD71/TER119 flow-cytometric assay. J. Vis. Exp. https://doi.org/ 10.3791/2809 (2011)

58. Moras, M., Lefevre, S. D. \& Ostuni, M. A. From erythroblasts to mature red blood cells: organelle clearance in mammals. Front. Physiol. 8, 1076 (2017).

59. Lamark, T. et al. Interaction codes within the family of mammalian Phox and Bem1p domain-containing proteins. J. Biol. Chem. 278, 34568-34581 (2003).

60. Sin, J. et al. Mitophagy is required for mitochondrial biogenesis and myogenic differentiation of C2C12 myoblasts. Autophagy 12, 369-380 (2016).

61. Liu, L. et al. Mitochondrial outer-membrane protein FUNDC1 mediates hypoxia-induced mitophagy in mammalian cells. Nat. Cell Biol. 14, 177-185 (2012).

62. Andres, A. M. et al. Mitophagy is required for acute cardioprotection by simvastatin. Antioxid. Redox Signal 21, 1960-1973 (2014).

63. Wilkinson, E. L., Sidaway, J. E. \& Cross, M. J. Statin regulated ERK5 stimulates tight junction formation and reduces permeability in human cardiac endothelial cells. J. Cell Physiol. 233, 186-200 (2018).

64. Baechler, B. L., Bloemberg, D. \& Quadrilatero, J. Mitophagy regulates mitochondrial network signaling, oxidative stress, and apoptosis during myoblast differentiation. Autophagy 15, 1606-1619 (2019).

65. Adrain, C., Creagh, E. M. \& Martin, S. J. Apoptosis-associated release of Smac/ DIABLO from mitochondria requires active caspases and is blocked by $\mathrm{BCl}-2$. EMBO J. 20, 6627-6636 (2001). 